Tohoku J. Exp. Med., 2007, 211, 347-352

\title{
High Serum Levels of Adiponectin Improve Coronary Collateral Development in Patients with Coronary Artery Disease
}

\author{
Serdar Soydinc, ${ }^{1}$ Vedat Davutoglu ${ }^{1}$ and Ibrahim Sari ${ }^{1}$ \\ ${ }^{1}$ Department of Cardiology, Gaziantep University, School of Medicine, Gaziantep, Turkey
}

Soydinc, S., Davutoglu, V. and SARI, I. High Serum Levels of Adiponectin Improve Coronary Collateral Development in Patients with Coronary Artery Disease. Tohoku J. Exp. Med., 2007, 211 (4), 347-352 — Adiponectin is a novel polypeptide that modulates endothelial function. Association between high serum adiponectin level and stimulation of new blood vessel formation have been reported in two experimental studies, however, data in humans are lacking. We sought to determine relationship between serum adiponectin and collateral vessel development in patients with coronary artery disease. We included 89 patients with stable angina pectoris and angiographically documented total occlusion in one of the major coronary arteries. Coronary collateral circulation was graded according to Rentrop scoring method in which collateralisation was graded between 0 and 3 from the poorest to the best. Adiponectin was determined by the ELISA method. High serum adiponectin level were significantly associated with increased new collateralisation ( $p=$ 0.001). With the increase of body mass index and waist circumference, the collateral development decreased ( $p=0.001, p=0.002$; respectively). Presence of the diabetes mellitus (DM) was more frequent in those with poor collateral group than in those with good collateral group. DM was associated with poor collateral development $(p=0.002)$. In multiple stepwise logistic regression analysis, low level of serum adiponectin $(p=0.0001)$, waist circumference $(p=0.001)$, and presence of DM $(p=0.003)$ were found to be significant independent predictors of poor collateral formation. In conclusion, we have shown for the first time that elevated levels of serum adiponectin are associated with coronary collateral development in patients with coronary artery disease and additionally, the present study confirms many of the metabolic associations reported previously with adiponectin. - adiponectin; coronary collateral development

(C) 2007 Tohoku University Medical Press

Improved survival of patients with acute coronary syndrome, has led to an increase in the number of patients with chronic coronary artery disease. In the setting of severe ischemia, the collateral artery development is important in respect of favorable effects on symptoms and functional class in these patients. Adiponectin is a novel polypeptide that is highly specific to adipose tissue (Shibata et al. 2004). It is present in human plasma and adipose tissue and is induced during adipogenesis. Adiponectin has been associated with the development of atherosclerotic vascular

Received December 21, 2006; revision accepted for publication February 26, 2007.

Correspondence: Vedat Davutoglu, M.D., Gaziantep University, School of Medicine, Departmant of Cardiology, 27310, Gaziantep, Turkey.

e-mail: davutoglu@gantep.edu.tr 
disease in some epidemiological studies (Ouchi et al. 2006). The notion that plasma adiponectin concentrations are lower in patients with coronary artery disease than in age- and body-mass indexadjusted control subjects supports the concept that adiponectin could somehow protect from atherosclerosis. In addition, it has been suggested that decreased plasma adiponectin concentrations in diabetes may be an indicator of macroangiopathy. Adiponectin modulates endothelial function and has an inhibitory effect on vascular smooth muscle cell proliferation.

Additionally it has antiinflammatory and antiatherogenic effects as well as multiple beneficial effects on metabolism (Ouchi et al. 2006). Recent two experimental studies have suggested that high serum adiponectin levels can function to stimulate new blood vessel formation (Ouchi et al. 2004; Shibata et al. 2004), however, data in humans are lacking. Accordingly, we conducted a study to assess the association between baseline serum adiponectin levels and collateral development, assessed by the Rentrop score, in patients with coronary artery disease who has one occluded coronary artery on coronary angiography.

\section{Methods}

\section{Study Patients}

Cases were selected among patients who underwent diagnostic coronary angiography for suspected coronary artery disease at Gaziantep University Hospital. The subjects were newly diagnosed 89 patients with stable angina pectoris and angiographically documented total occlusion in one of the major coronary arteries. Demographic characteristics, presence of prior myocardial infarction, conventional risk factors for coronary artery disease and medications were recorded by trained physicians. Because stenosis in the donor artery can affect collateral formation (Fujita et al. 1991), patients with significant stenosis in the donor artery and patients with significant multivessel coronary artery disease were excluded. Additionally, since the degree of stenosis in the collateral recipient artery may also affect the collateral development (Cohen et al. 1989) only the patients with total occlusion of one major coronary artery were included in the study. The patients who underwent previous revascularization procedures or who has history of myocardial infarction (MI) within the last 3 months were excluded from the study. Patients with statin and angiotensin converting enzyme inhibitor (ACEI) treatment/ angiotensin receptor blocker (ARB) treatment, with acute or chronic inflammatory disease, carotid or peripheral arterial disease, malignancies, renal or thyroid diseases, heart failure, vascular disease, or hepatic dysfunction (viral and nonviral, transaminases more than 3-fold the normal value) were also excluded from the study, as these conditions can influence the evaluation of collateral circulation. Patients with systolic or diastolic blood pressure $>140 \mathrm{mmHg}$ or $90 \mathrm{mmHg}$ or who were under antihypertensive treatment were considered to have hypertension (HT). Patients who were being treated for diabetes mellitus (DM) or who had symptoms of DM and a fasting glucose concentration more than $126 \mathrm{mg} / \mathrm{dl}$ were considered to have DM. None of the patients was receiving hormone replacement therapy.

\section{Coronary angiography}

Coronary angiography was performed via the femoral artery using Judkins technique (Integris H 5000, Philips Medical Systems, Netherland). Significant coronary narrowing was defined as stenosis of $>50 \%$ of at least one main branch of the coronary arteries. Coronary collateral circulation was graded according to the Rentrop score as follows: Grade 0: no filling of any collateral vessels; Grade 1: filling of side branches of the artery to be perfused by collateral vessels without visualization of the epicardial segment; Grade 2: partial filling of the epicardial artery by collateral vessels; Grade 3: complete filling of the epicardial artery by collateral vessels (Cohen and Rentrop 1986). Patients were grouped according to Rentrop collateral development score. The ethics committee of Gaziantep University Hospital approved this study and informed consent was obtained from each patient.

Laboratory measurements were made blind to participants' disease status, with samples from patients randomly distributed among assay plates. Adiponectin (ng/ $\mathrm{ml}$ ) was determined using a validated sandwich ELISA employing an adiponectin-specific monoclonal and polyclonal antibody (RayBiotech Inc., Norcross, GA, USA), which has been demonstrated by Arita et al. (Arita et al. 1999). For the serum measurement of adiponectin, we stored serum samples without adding anticoagulation agent. Blood was drawn in the morning after an overnight fast and serum concentration of total cholesterol, triglyceride, and high density lipoprotein- cholesterol (HDL-C) were measured. 
Statistical analysis

The Statistical Package for the Social Science for Windows 10.0 (SPSS Inc., Chicago, IL, USA) was used for statistical analysis. Categorical variables were compared by chi-square analysis. Relationships between variables were tested by Pearson and Spearman correlations. Logistic regression analysis was performed to evaluate the independent effects of the distinct variables to collateral development. We calculated $95 \%$ confidence intervals for the odds ratios. $P$ values $<0.05$ were considered statistically significant.

\section{RESUlts}

A comparison of the baseline clinical characteristics between four Rentrop classes is summarized in Table 1 . The study population consisted of 89 patients with documented total occlusion in one of the coronary arteries. The four groups were similar in terms of age, sex, family history, history of coronary artery disease, prior MI, systemic hypertension, smoking, low density lipoprotein (LDL) cholesterol level, HDL cholesterol level. Correlation analysis shows significant correlation beween adiponectin concentration and
Rentrop score; thus, high serum adiponectin level was significantly associated with increased new collateralisation ( $p=0.001, \mathrm{r}=0.642)$ (Fig. 1.). With the increase of body mass index and waist circumference, the collateral development decreased $(\mathrm{r}=-0.336, p=0.001 ; \mathrm{r}=-0.476$, $p=0.002$; respectively). Presence of the DM was more frequent in those with poor collateral group than in those with good collateral group. Presence of the DM was significantly more frequent in those with poor collateral group than in those with good collateral group. DM was associated with poor collateral development $(p=0.002, \mathrm{r}=0.354)$. In a multiple stepwise logistic regression analysis, low level of adiponectin concentration (odds ratio $[\mathrm{OR}]=4.39,95 \%$ confidence interval $[\mathrm{CI}]=1.83$ $-11.79, p=0.0001)$, waist circumference $(\mathrm{OR}=$ $3.27,95 \% \mathrm{CI}=1.43-13.69, p=0.001)$, and presence of $\mathrm{DM}(\mathrm{OR}=2.37,95 \% \mathrm{CI}=1.63-9.48$, $p=0.003$ ) were found to be significant independent predictors of angiographically determined poor collateral formation.

TABLE 1. Baseline characteristics of patients by Rentrop collateral classification.

\begin{tabular}{lccccc}
\hline & $\begin{array}{c}\text { Rentrop } 0 \\
n=8\end{array}$ & $\begin{array}{c}\text { Rentrop 1 } \\
n=22\end{array}$ & $\begin{array}{c}\text { Rentrop 2 } \\
n=25\end{array}$ & $\begin{array}{c}\text { Rentrop 3 } \\
n=34\end{array}$ & $p$ values \\
\hline Age (years) & $61.9 \pm 10.8$ & $58.0 \pm 10.1$ & $59.3 \pm 12.0$ & $60.0 \pm 11.0$ & NS \\
Gender (Male/Female) & $5 / 3$ & $14 / 8$ & $16 / 9$ & $21 / 13$ & NS \\
& $(62.5 \% / 37.5 \%)$ & $(63.6 \% / 36.4 \%)$ & $(64 \% / 36 \%)$ & $(62 \% / 38 \%)$ & \\
Family history of heart & $3(37.5 \%)$ & $7(31.7 \%)$ & $8(32 \%)$ & $10(29 \%)$ & NS \\
disease & & & & & \\
Prior MI & $3(37.5 \%)$ & $7(31 \%)$ & $8(32 \%)$ & $13(38 \%)$ & NS \\
Diabetes mellitus & $3(37.5 \%)$ & $10(45.5 \%)$ & $6(24 \%)$ & $5(14 \%)$ & 0.002 \\
Systemic hypertension & $3(37.5 \%)$ & $10(45 \%)$ & $11(44 \%)$ & $12(35 \%)$ & NS \\
Smoking & $3(37.5 \%)$ & $7(32 \%)$ & $8(32 \%)$ & $13(38 \%)$ & NS \\
Body mass index $\left(\mathrm{kg} / \mathrm{m}^{2}\right)$ & $28.75 \pm 3.11$ & $29.66 \pm 3.99$ & $28.06 \pm 4.84$ & $24.41 \pm 2.41$ & 0.001 \\
Waist circumference & $98.25 \pm 7.2$ & $99.8 \pm 9.7$ & $97.5 \pm 13.7$ & $86.15 \pm 8.0$ & 0.001 \\
Total cholesterol $(\mathrm{mg} / \mathrm{dl})$ & $174.75 \pm 41.8$ & $191.31 \pm 61.6$ & $167.96 \pm 35.78$ & $155.17 \pm 43.5$ & 0.05 \\
LDL cholesterol $(\mathrm{mg} / \mathrm{dl})$ & $115.6 \pm 32.8$ & $110.9 \pm 49.8$ & $98.8 \pm 32.8$ & $95.1 \pm 45.0$ & $\mathrm{NS}$ \\
Triglycerides $(\mathrm{mg} / \mathrm{dl})$ & $153.0 \pm 82.5$ & $216.2 \pm 110.0$ & $167 \pm 77.8$ & $141 \pm 57.45$ & 0.01 \\
HDL cholesterol $(\mathrm{mg} / \mathrm{dl})$ & $32.58 \pm 11.25$ & $38.78 \pm 11.42$ & $34.70 \pm 8.5$ & $36.36 \pm 1.0$ & $\mathrm{NS}$ \\
Ejection Fracton $(\%)$ & $47.0 \pm 7.1$ & $45.0 \pm 8.4$ & $47.0 \pm 9.5$ & $48.0 \pm 7.8$ & NS \\
\hline
\end{tabular}

NS, non-significant, MI, myocardial infarction; LDL, low density lipoprotein; HDL, high density lipoprotein. 


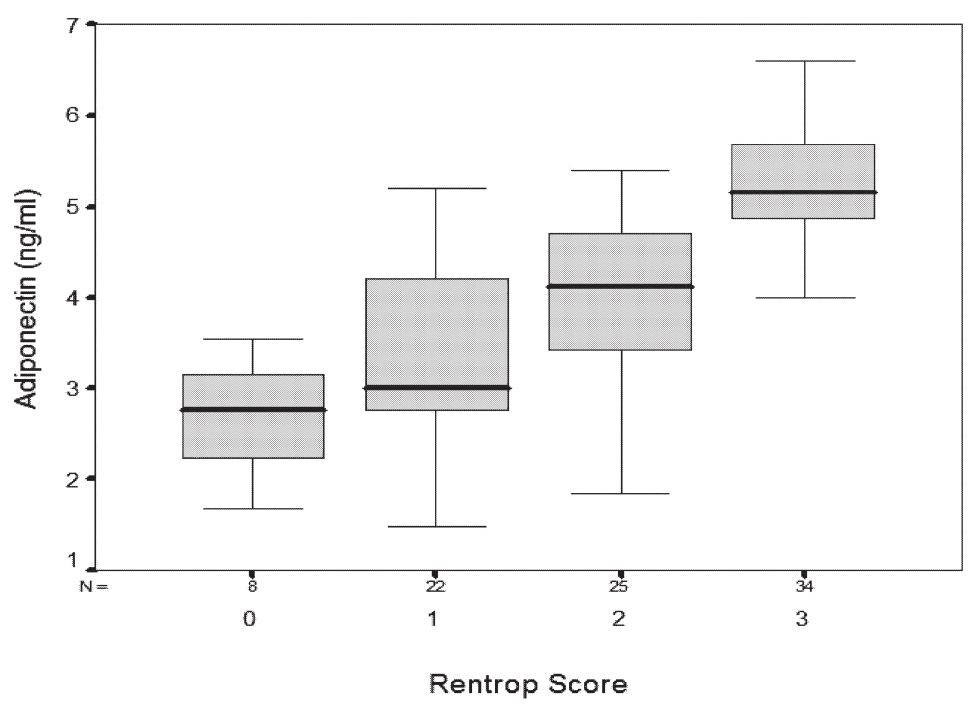

Fig. 1. Strong interrelationship between serum adiponectin levels (ng/ml) and coronary collateral development which is represented with Rentrop score is shown in the figure. High serum level of adiponectin is associated with well developed new vessel formation. $\mathrm{N}$, number of patients in each group.

\section{Discussion}

The main finding of this study is that the patients with higher adiponectin level are strongly associated with the presence of angiographically visible collateral vessels than patients who has low level of adiponectin concentration. Our findings, therefore, support recent two experimental reports in which adiponectin stimulated new blood vessel formation (Ouchi et al. 2004; Shibata et al. 2004). In one of these studies, Ouchi et al. (2004) showed that adiponectin promotes proangiogenic cellular responses in endothelial cells. Thus identifies the promotion of blood vessel growth as a new role for the adiponectin. They proposed that the ability of adiponectin to stimulate angiogenesis is likely due, at least in part, to its ability to promote endothelial cell migration and stimulate the differentiation of these cells into capillary-like structures. Furthermore, they suggested that adiponectin might function to stimulate the new blood vessel growth by promoting cross-talks between adenosine monophosphateactivated protein kinase and Akt signaling within endothelial cells.

In addition, our findings demonstrate that $\mathrm{DM}$ and increase in waist circumference also impairs the collateral formation. Thus, in this study we confirm some of the metabolic associations reported previously with adiponectin (Shimada et al. 2004). In this regard, our findings are in accord with the literature in this respect.

Indeed, the collateral circulation is the most effective natural defense mechanism of the diseased heart. Studies in experimental animals and humans have confirmed the protective role of collaterals against myocardial ischemia, myocardial infarction, and cell death (Williams et al. 1976). Even after myocardial infarction, collateral blood flow significantly improves recovery of the function of the infarct zone (Epstein 1988). Very little is known about the triggers and mediators of collateral artery growth in ischemic vascular diseases. The mechanical effects of increased blood flow previously have been hypothesized to play a major role. Collateral arteries develop rapidly following chronic occlusion of a major coronary artery, and collaterals play a critical role in reestablishing and maintaining blood flow to the ischemic myocardium (Schaper 1991).

Adiponectin has antiinflammatory and antiatherogenic effects as well as multiple beneficial effects on metabolism, in addition, adiponectin modulates endothelial function and has an inhibi- 
tory effect on vascular smooth muscle cell proliferation (Ukkola and Santaniemi 2002). Experimental evidence suggests that adiponectin might play a protective role against atherosclerosis. Low serum adiponectin levels have been reported in coronary artery disease, as well as associated with some risk factors of cardiovascular disease such as male sex, high blood pressure, obesity and type 2 DM (Arita et al. 1999; Ouchi et al. 1999; Kazumi et al. 2002). Importantly, our study findings propose a new additive protective mechanism by the enhancement of collateralisation by means of adiponectin.

The agents which have the potential of increasing adiponectin levels are peroxisome proliferator-activated receptor (PPAR) $\gamma$ agonists (Maeda et al. 2001; Combs et al. 2002). Treatment with rosiglitazone, a PPAR $\gamma$ agonist, increased circulating adiponectin levels (Yang et al. 2002). Importantly, we think that the application of the thiazolidinediones therapies to increase adiponectin in the ischemic heart which also may increase collateralisation may be useful to increase circulation in the myocardium at ischemic risk. Further investigations in patients with the hypoadiponectinemic situations are required to clarify these aspects of the potential therapeutic applications of this adipocytokine.

Recently a major epidemic study revealed that central obesity, as measured with waist circumference, is a primary factor affecting levels of circulating adiponectin. (Steffes et al. 2004). Our study is in accord with this study. It is well known that weight loss and regular activities have favorable effect on waist circumferenence and body weight, thus may result in increased adiponectin level.

Taken together, well developed coronary collateral arteries in patients with coronary artery disease mitigate myocardial infarcts with less ventricular aneurysm formation and improved ventricular function, reduce future cardiovascular events, and improve survival.

Therapeutic angiogenesis/arteriogenesis are new strategies for revascularising ischemic myocardial tissue by formation of "natural bypasses" that is, collateral vessels. Furthermore, since there are many patients with intractable angina pectoris who are not candidates for percutaneous coronary intervention and coronary bypass surgery, searching for additional treatment options for increasing adiponectin levels for these patients is of great importance in respect of angiogenesis. In our view, therapeutic promotion of collateral enhancement by the adiponectin therapies appears to be a valuable treatment strategy in those patients and it seems to play an additional protective role in patients with chronic heart disease. The effect of adiponectin on prognosis of coronary artery disease merits further study.

\section{Limitations}

There are several limitations in the present study. The major limitation of this study is the patients' selection bias. The conclusions of this study are derived from patients with total coronary artery occlusion. Therefore, the significance of collateral circulation is limited to this group of patients. We should not extend our study results to broader populations of patients with myocardial infarction. The second limitation is related to the angiographic evaluation of coronary arteries. In the present study, collateral vessel formation was assessed using angiographic criteria. Angiographically visible collaterals represent only a fraction of the total collateral vessels because collaterals are angiographically demonstrable only when they reach 200 micrometers (Gibson et al. 1999). Therefore, patients characterized as lacking collateral circulation might have small collaterals. The definition of DM was determined by fasting glucose level. The prevalence of diabetes is possibly underestimated in this study, since the data of $\mathrm{HbA} 1 \mathrm{c}$ and/or $75 \mathrm{~g}$ oral glucose tolerance test was not available.

\section{Clinical implications}

To our knowledge the present study is the first to demonstrate the strong association between adiponectin and collateral development clinically. Our findings raise the question of whether treatment strategies targeting adiponectin rising therapy may improve collateral formation. However, our study is cross-sectional and therefore we can- 
not comment on a causal relationship. This needs to be assessed in further studies.

\section{Conclusion}

In conclusion, in the present study we confirm many of the metabolic associations reported previously with adiponectin. Addionally we have shown for the first time that elevated levels of adiponectin are associated with coronary collateral development in patients with coronary artery disease.

\section{Acknowledgments}

We thank Dr Emre Cagliyan and Dr Ahmet Celik for patients data and blood sample collection.

\section{References}

Arita, Y., Kihara, S., Ouchi, N., Takahashi, M., Maeda, K., Miyagawa, J., Hotta, K., Shimomura, I., Nakamura, T., Miyaoka, K., Kuriyama, H., Nishida, M., Yamashita, S., Okubo, K., Matsubara, K., Muraguchi, M., Ohmoto, Y., Funahashi, T. \& Matsuzawa, Y. (1999) Paradoxical decrease of an adipose-specific protein, adiponectin, in obesity. Biochem. Biophys. Res. Commun., 257, 79-83.

Cohen, M. \& Rentrop, K.P. (1986) Limitation of myocardial ischemia by collateral circulation during sudden controlled coronary artery occlusion in human subjects: a prospective study. Circulation, 75, 325-334.

Cohen, M., Sherman, W., Rentrop, K.P. \& Gorlin, R. (1989) Determinants of collateral filling observed during sudden controlled coronary artery occlusion in human subjects. $J$. Am. Coll. Cardiol., 13, 297-303.

Combs, T.P., Wagner, J.A., Berger, J., Doebber, T., Wang, W.J., Zhang, B.B., Tanen, M., Berg, A.H., O'Rahilly, S., Savage, D.B., Chatterjee. K., Weiss, S., Larson, P.J., Gottesdiener, K.M., Gertz, B.J., Charron, M.J., Scherer, P.E. \& Moller, D.E. (2002) Induction of adipocyte complement-related protein of 30 kilodaltons by PPAR gamma agonists: a potential mechanism of insulin sensitization. Endocrinology, 143, 998-1007.

Epstein, S.E. (1988) Influence of stenosis severity on coronary collateral development and importance of collaterals in maintaining left ventricular function during acute coronary occlusion. Am. J. Cardiol., 61, 866-868.

Fujita, M., McKown, D.P., McKown, M.D. \& Franklin, D. (1991) Effects of stenosis of donor arteries on collateral flow and regional myocardial function in conscious dogs with well-developed coronary collateral circulation. Coron. Artery Dis., 2, 815-822.

Gibson, C.M., Ryan, K., Sparano, A., Moynihan, J.L., Rizzo, M., Kelley, M., Marble, S.J., Laham, R., Simons, M.,
McClusky, T.R. \& Dodge, J.T., Jr. (1999) Angiographic methods to assess human coronary angiogenesis. Am. Heart. J., 137, 169-179.

Kazumi, T., Kawaguchi, A., Sakai, K., Hirano, T. \& Yoshino, G. (2002) Young men with high-normal blood pressure have lower serum adiponectin, smaller LDL size, and higher elevated heart rate than that with optimal blood pressure. Diabetes Care, 25, 971-976.

Maeda, N., Takahashi, M., Funahashi, T., Kihara, S., Nishizawa, H., Kishida, K., Nagaretani, H., Matsuda, M., Komuro, R., Ouchi, N., Kuriyama, H., Hotta, K., Nakamura, T., Shimomura, I. \& Matsuzawa, Y. (2001) PPAR gamma ligands increase expression and plasma concentrations of adiponectin, an adipose-derived protein. Diabetes, 50, 2094-2099.

Ouchi, N., Kihara, S., Arita, Y., Maeda, K., Kuriyama, H., Okamoto, Y., Hotta, K., Nishida, M., Takahashi, M., Nakamura, T., Yamashita, S., Funahashi, T. \& Matsuzawa, Y. (1999) Novel modulator for endothelial adhesion molecules: adipocyte- derived plasma protein adiponectin. Circulation, 100, 2473-2476.

Ouchi, N., Kobayashi, H., Kihara, S., Kumada, M., Sato, K., Inoue, T., Funahashi, T. \& Walsh, K. (2004) Adiponectin Stimulates Angiogenesis by Promoting Cross-talk between AMP-activated Protein Kinase and Akt Signaling in Endothelial Cells. J. Biol. Chem., 279, 1304-1309.

Ouchi, N., Shibata, R. \& Walsh, K. (2006) Cardioprotection by Adiponectin. Trends. Cardiovasc. Med., 16, 141-146.

Schaper, W. (1991) Angiogenesis in the adult heart. Basic. Res. Cardiol., 86, 51-56.

Shibata, R., Ouchi, N., Kihara, S., Sato, K., Funahashi, T. \& Walsh, K. (2004) Adiponectin Stimulates Angiogenesis in Response to Tissue Ischemia through Stimulation of AMPactivated Protein Kinase Signaling. J. Biol. Chem., 279, 28670-28674.

Shimada, K., Miyazaki, T. \& Daida, H. (2004) Adiponectin and atherosclerotic disease. Clinica Chimica Acta., 344, 1-12.

Steffes, M.W., Gross, M.D., Schreiner, P.J., Yu, X., Hilner, J.E., Gingerich, R. \& Jacobs, D.R., Jr. (2004) Serum Adiponectin in Young Adults - Interactions with CentralAdiposity, Circulating Levels of Glucose, and Insulin Resistance: The CARDIA study. Ann. Epidemiol., 14, 492-498.

Ukkola, O. \& Santaniemi, M. (2002) Adiponectin: a link between excess adiposity and associated comorbidities? $J$. Mol. Med., 80, 696-702.

Williams, D.O., Amsterdam, E.A., Miller, R.R. \& Mason, D.T. (1976) Functional significance of coronary collateral vessels in patients with acute myocardial infarction: Relation to pump performance, cardiogenic shock and survival. Am. J. Cardiol., 37, 345-351.

Yang, W.S., Jeng, C.Y., Wu, T.J., Tanaka, S., Funahashi, T., Matsuzawa, Y., Wang, J.P., Chen, C.L., Tai, T.Y. \& Chuang, L.M. (2002) Synthetic peroxisome proliferator-activated receptor-gamma agonist, rosiglitazone, increases plasma levels of adiponectin in type 2 diabetic patients. Diabetes Care, 25, 376-380. 